\title{
Combinatorial Discrete Choice *
}

\author{
Costas Arkolakis \\ Yale University and NBER \\ Fabian Eckert \\ Yale University \\ First Version: January 2017 \\ PRELIMINARY
}

\begin{abstract}
Combinatorial problems are prevalent in economics but the large dimensionality of potential solutions substantially limits the scope of their applications. We define and characterize a general class that we term combinatorial discrete choice problems and show that it incorporates existing problems in economics and engineering. We prove that intuitive sufficient conditions guarantee the existence of simple recursive procedures that can be used to identify the global maximum. We propose such an algorithm and show how it can be used to revisit problems whose computation was deemed infeasible before. We finally discuss results for a class of games characterized by these sufficient conditions.
\end{abstract}

${ }^{*}$ We thank Jake DiCicco, Tra Nguyen, Andrew Salmon, Benjamin Tong, Yunus Tuncbilek for outstanding research assistance amd input through various stages of this project. We also thank Chrysanthos Gounaris for early conversations on the topic as well as David Atkin, Arnaud Costinot, Dave Donaldson, Penny Goldberg, and Samuel Kortum for their comments and suggestions. All errors are our own. 


\section{Introduction}

Discrete choice combinatorial problems are ubiquitous in economics. Across fields such as Consumer Theory, Industrial Organization, and International Trade researchers employ discrete choice formulations to model consumer choice problems, input decisions and plant location choices with positive or negative complementarities. Complementarities make decisions interdependent leading to a potential solution space that grows exponentially in the number of choices. This makes solving these problems very hard or even computationally infeasible. ${ }^{1}$

We show that the same complementarities that dissuaded economists from these problems are key to handling combinatorial optimization in discrete choice. For our characterization we define a general class of problems that we term combinatorial discrete choice problems, that includes well known problems and models in economics and engineering. In these problems the agent (firm, consumer, etc) maximizes a return function that is defined over an $n$-dimensional Boolean domain representing the multiple choices that the agents may make.

The key restriction that we impose is one of increasing or decreasing loose differences of the return function. This restriction is intuitive: in case of the loose increasing difference condition, if the marginal value of a coordinate $i$ is positive for a given choice vector, it has to remain so even as more entries of the vector are switched to 1 . The intuition for the loose decreasing difference condition is analogous. Supermodularity of the return function is a sufficient condition for it exhibiting loose increasing differences while the submodularity property is sufficient for decreasing differences it is submodularity). Both conditions are of wide theoretical and practical use in economics in all contexts in which complementarities between choices are important.

To solve this class of problems we introduce a new iterative mapping defined on a Boolean lattice. Based on this iterative mapping we present three key results that provide a characterization of the optimization of this class of combinatorial discrete choice problems, when increasing or decreasing loose differences hold.

The first two results, that correspond to Theorems 1 and 2 guarantee the existence of simple recursive procedure that can be used to identify the global maximum. Theorem 1 implies that iterating our mapping on a Boolean lattice will always converge to a subset of the potential solution space that contains the optimizing vector under the sole condition that the return function exhibits the loose monotonic differences property. Theorem 1 can

\footnotetext{
${ }^{1}$ Combinatorial problems are typically NP-hard (eg. in the Boolean domain: $2^{N}$ combinations). In addition, a well-known theorem from combinatorial optimization implies that without additional structure no solution method can perform better than random search (see no free-lunch Theorem Wolpert and Macready (1997)).
} 
then be viewed as method to reduce the set of potentially optimizing vectors by exploiting the structure provided by the loose monotonic difference assumption. The basic intuition is that by evaluating the objective function at points of maximal complementarities and exploiting the monotonicity assumption many potential solutions can usually be excluded without having to evaluate them. While the resulting set contains the optimum it may still be so large that evaluating the objective function at all of its elements is infeasible. Theorem 2 illustrates that an extension of this algorithm that applies it repeatedly by splitting the lattices where the choice is ambiguous always delivers the unique maximizing vector.

We finally discuss results for a class of games characterized by these sufficient conditions. To do so, we define a new class of games, which we term loose monotonic games in which the payoff function in which the the payoff function of each player exhibits either loose increasing or decreasing differences while the joint strategy space features decreasing differences. A special case of this are games in which the payoff function of one player is submodular in own strategies and there are decreasing differences in the joint strategy space of the players. We term such games "submodular" games. A pure strategy Nash equilibrium can be shown to exist in all loose monotonic games. In fact, our algorithm is shown to always identify such an equilibrium point.

An array of papers in Industrial Organization and International Trade present models that involve the solution of combinatorial discrete choice problems. In Industrial Organization both Holmes (2011) and Jia (2008) develop models in which a chain store needs to make an entry decision across a multitude of spatially distributed markets and where market specific profits are interdependent. In particular Holmes (2011) models business stealing effects between different chain stores branches, while Jia (2008) highlights positive spillovers from economies of scale. Hendel (1999) is an early paper formulating a "multiple discrete choice" problem pertaining to the optimal number of computers per brand to be purchased by a firm. In international trade a range of new papers studying the optimal choice of production plants across countries naturally involve combinatorial discrete choice problems. Arkolakis et al. (2013) and Tintelnot (2016) are examples. Antras et al. (2014) model the optimal input choices of a firm from a menu of international suppliers as a combinatorial discrete choice problem. In addition a nascent literature on global value chains formulates firm problems that can be written as combinatorial discrete choice problems such as in Antràs and de Gortari (2017). Morales et al. (2015) features a model of the export choice of firms that becomes a combinatorial one once some of the usual gravity assumptions are relaxed. ${ }^{2}$

\footnotetext{
${ }^{2}$ See also Carvalho and Voigtländer (2014) who demonstrate how the evolution of a network and in particular the creation of new links within a network can be though of a as a discrete choice problem with interrelated payoffs.
} 
A procedure developed in Jia (2008) constitutes the only solution method for the class of combinatorial discrete choice problems that appears in the above papers. The method in this paper nest and extends the approach introduced in Jia (2008). We formally show that the procedure in Jia (2008) applies to functions that exhibit loose increasing differences. We then introduce a new technique that extends Jia (2008) to functions that satisfy the converse property of loose decreasing differences. Finally, we present an iterative application of our solution algorithm that allows to identify the maximizing vector of any loose monotonic function at all times. Similar to Jia (2008) our technique exploits results from lattice theory in particular a theorem first proved by Knaster (1928) and later generalized by Tarski (1955). A typical alternative to actually solving the combinatorial optimization problem is to use a moment inequality approach to estimate the parameters of the return function and only evaluate a few of the possible combinations to estimate bounds for the parameters. Pakes et al. (2015), Holmes (2011) and Morales et al. (2015) are examples of this approach. ${ }^{3}$

Our paper also relates to a large literature on supermodular games discussed by Topkis (1979), Vives (1990), Milgrom and Shannon (1994) and Zhou (1994). In particular we define a class of $n$-market $m$-player aggregative games on a lattice that we refer to as loose monotonic games and where the individual firm solves a combinatorial discrete choice problem. Conditional on the functional form restriction we impose these games are more general than supermodular games: loose monotonic games can have firm profit functions that are either supermodular or submodular in the own strategy while exhibiting decreasing differences in the joint strategy space. ${ }^{4}$ To the best of our knowledge there is no paper that has formally treated "submodular games", that is games where players are competing in an entry game across several markets, while also facing internal negative spillovers between markets for example due to cannibalization. ${ }^{5}$ Results by Voorneveld (2000) and Acemoglu and Jensen (2013) guarantee the existence of pure strategy equilibria in such games and demonstrate how our techniques can be used to always locate such an equilibrium point. ${ }^{6}$

Lastly, our paper is related to a literature on Pseudo-Boolean optimization in Operations Research detailed in Boros and Hammer (2002). This literature is occupied with maximizing integer valued multi-varied objectives where individual decisions are interdependent. A canonical problem is the Simple Plant Location problem (SPLP) surveyed in Balinski (1964)

\footnotetext{
${ }^{3}$ A key drawback of moment inequality approaches is that they do not allow for counterfactual analysis which requires the explicit solution of the model.

${ }^{4}$ By joint strategy space we refer to the space mapped out by the collection of strategy vectors by all players.

${ }^{5}$ Consider the situation of Walmart: distinct branches of the chain do compete with business when closely located to one another (see eg. Holmes (2011)), while it also competes with other chains such as Kmart (see eg. Jia (2008)).

${ }^{6}$ In particular, the supermodular multi-market entry game in Jia (2008) is a nested in our setup.
} 
or Owen and Daskin (1998). We demonstrate how SPLP problems can be understood as submodular combinatorial discrete choice problems that can be solved using our solution technique.

In the next section we introduce the general problem set up and introduce the necessary and sufficient condition that guarantees the existence of an effective solution procedure. In section 3 we state our main theorems pertaining to the maximization of combinatorial discrete choice problems. Section 4 discusses a class of related games and demonstrates how our maximization procedure can be used to solve for related pure strategy Nash equilibria. Section 5 concludes.

\section{Combinatorial Discrete Choice Problems}

In this section we introduce the class of discrete choice problems that are the subject of this paper and which we term combinatorial discrete choice problems. We then discuss the key restriction on the objective function in such problems that greatly simplifies the computation of their solution. Lastly, we discuss the prevalence of combinatorial discrete choice problems in the economics literature that naturally satisfy our key restriction.

\subsection{Combinatorial Problems: General Setup}

We consider the problem of maximizing a real valued function $\Pi$ defined on a $n$-dimensional Boolean domain $\mathcal{B}^{n}=\{0,1\}^{n}:{ }^{7}$

$$
\Pi: \mathcal{B}^{n} \rightarrow \mathbb{R}
$$

The objective is to find the maximizing vector $I \in \mathcal{B}^{n}$, denoted $I^{\star}$, such that

$$
I^{*}=\underset{I \in \mathcal{B}^{n}}{\arg \max } \Pi(I) .
$$

This class of real-valued discrete choice problems over Boolean vectors has a variety of applications in economics and other sciences. The particular challenge of these problems is that individual decisions are typically not independent. ${ }^{8}$ It is this interdependence across decisions that makes these problems combinatorial and inflates the number of potential

\footnotetext{
${ }^{7}$ Functions of the form $\Pi: \mathcal{B}^{n} \rightarrow \mathbb{R}$ are known as pseudo-Boolean functions in mathematics. In particular applications $\Pi$ could be utility, profits, etc. A Boolean function is a function that maps a Boolean space into Boolean space. Boolean functions are a subset of Pseudo Boolean functions since $\mathcal{B}^{n} \subset \mathbb{R}^{n}$.

${ }^{8}$ Intuitively, the cross derivative of $\Pi(\cdot)$ with respect to two different coordinates of the vector $I$ is typically not zero. The concept of a derivative of a pseudo-Boolean function is defined more rigorously below.
} 
solutions to $2^{n}$. This dimensionality, in turn, makes the solution of these problems infeasible to compute even on high powered computers. ${ }^{9}$

Unfortunately, a well known theorem in computational mathematics (see for example, Wolpert and Macready (1997)), the so-called 'no-free-lunch' theorem states that without further restriction on the properties of the pseudo-Boolean objective, $\Pi$, one cannot beat random search algorithms in identifying $I^{*}$. Combinatorial discrete choice problems in economics often naturally impose a restriction on the objective function that allows researchers to improve upon random search. Such restriction and the associated solution method are the subject of this paper.

\subsection{Loose Monotonic Combinatorial Problems}

Here we first introduce some further notational conventions. For any $I, I^{\prime} \in \mathcal{B}^{n}$, we say that $I \geq I^{\prime}$ if $I_{i} \geq I_{i}^{\prime}$ for all $i=1, \ldots$, n. ${ }^{10}$ Also denote by $I^{i \rightarrow 1}$ the Boolean vector $I$ with the $i$ th coordinate set to 1 . We can then define the derivative of a function on the Boolean domain with respect to the $i$ th coordinate as follows: $D_{i} \Pi(I)=\Pi\left(I^{i \rightarrow 1}\right)-\Pi\left(I^{i \rightarrow 0}\right)$. We will also refer to this as the marginal value of the $i$ th coordinate. With this notation in hand we can introduce the key restriction we impose on the Boolean return function in what follows:

Definition. A function $f: \mathcal{B}^{n} \longrightarrow \mathbb{R}$ exhibits the loose increasing differences property if, for any $I, I^{\prime} \in \mathcal{B}^{n}$ such that $I \leq I^{\prime}$ we have

$$
\begin{aligned}
& D_{i}(f(I)) \geq 0 \Longrightarrow D_{i}\left(f\left(I^{\prime}\right)\right) \geq 0 \\
& D_{i}(f(I))>0 \Longrightarrow D_{i}\left(f\left(I^{\prime}\right)\right)>0 .
\end{aligned}
$$

A function $f: \mathcal{B}^{n} \longrightarrow \mathbb{R}$ exhibits the loose decreasing differences property if, for any $I, I^{\prime} \in$ $\mathcal{B}^{n}$ such that $I \leq I^{\prime}$ we have

$$
\begin{aligned}
& D_{i}(f(I)) \geq 0 \Longleftarrow D_{i}\left(f\left(I^{\prime}\right)\right) \geq 0 \\
& D_{i}(f(I))>0 \Longleftarrow D_{i}\left(f\left(I^{\prime}\right)\right)>0 .
\end{aligned}
$$

We will refer to functions satisfying either one of these conditions as functions exhibiting loose monotonic differences. The loose monotonic differences property is the necessary and

\footnotetext{
${ }^{9}$ The exponential growth of the potential solution space is known as the combinatorial explosion problem in combinatorics. In addition many problems of this sort can be shown to be NP-hard, meaning they cannot generally be proven to be solvable in less than polynomial time.

${ }^{10}$ The order relation "entry-wise comparison" establishes only a partial order on the $n$-dimensional Boolean lattice. It can easily be shown that $\mathcal{B}^{n}$ along with "entry-wise comparison" is a particular sort of partially ordered set known as lattice.
} 
sufficient condition for all our results below.

This restriction is intuitive: in case of the loose increasing difference condition, if the marginal value of a coordinate $i$ is positive for a given $I$ vector, it has to remain so even as more entries of $I$ are switched to 1 . More concretely if $I_{i}=1$ corresponds to opening a production site in location $i$, this means that if profitable for a given set of open production sites, this production site has to remain profitable even as more production sites are added to the initial set. The intuition for the loose decreasing difference condition is analogous.

On a continuous domain the loose monotonic difference condition corresponds to a restriction on the cross derivatives of the return function with respect to coordinate $i$ and all other coordinates $i^{\prime} \neq i$ of the $I$ vector. In the loose increasing differences case if the derivative with respect to the $i$ th coordinate is positive, the derivative of this with respect to other entries $i^{\prime}$ for which $I_{i^{\prime}}=0$, cannot be too negative. In particular it cannot be so negative as to turn the derivative of $\Pi$ with respect to $i$ negative as successive other coordinates are switched from $I_{i^{\prime}}=0$ to $I_{i^{\prime}}=1$.

It turns out that the loose monotonic differences condition is naturally satisfied by many economic models and we list prominent examples below and in more detail in the appendix. The assumption is also often easily verified in empirical applications: for example if a chain store opens more and more stores over the years and rarely closes existing ones the loose increasing difference assumption would seem to hold approximately for its underlying profit function.

A sufficient condition for the loose monotonic difference condition to hold for the return function $\Pi$ is for it to exhibit either the supermodularity or submodularity property. In what follows for any two elements $I, I^{\prime} \in \mathcal{B}^{n}$ we denote by $\max \left(I, I^{\prime}\right)$ the vector with the $i$ th component $\max \left(I_{i}, I_{i}^{\prime}\right)$ and by $\min \left(I, I^{\prime}\right)$ the vector with the $i$ th component $\min \left(I_{i}, I_{i}^{\prime}\right)$. We refer to $\max \left(I, I^{\prime}\right)$ as the least upper bound and to $\min \left(I, I^{\prime}\right)$ as the greatest lower bound of the pair $\left(I, I^{\prime}\right)$. We can then define:

Definition. A function $f: \mathcal{B}^{n} \longrightarrow \mathbb{R}$ is supermodular if it satisfies for all $I, I^{\prime} \in \mathcal{B}^{n}$

$$
f\left(\max \left(I, I^{\prime}\right)\right)-f(I) \geq f\left(I^{\prime}\right)-f\left(\min \left(I, I^{\prime}\right)\right)
$$

A function $f: \mathcal{B}^{n} \longrightarrow \mathbb{R}$ is submodular if it satisfies for all $I, I^{\prime} \in \mathcal{B}^{n}$,

$$
f\left(\max \left(I, I^{\prime}\right)\right)-f(I) \leq f\left(I^{\prime}\right)-f\left(\min \left(I, I^{\prime}\right)\right)
$$

We find it useful to introduce this sufficient condition since the properties of superand to a lesser degree submodular return functions is already well-established in economics 
and their relevant applications are numerous. The mathematical concepts of super- and submodularity formalize the notion of positive and negative complementarities so prevalent in economics. On a continuous domain these properties correspond to the cross-derivative of the return function with respect to coordinate $i$ and $i^{\prime}$ being either positive or negative over the whole domain, which provides an intuition for these properties being sufficient for the loose monotonic difference condition. ${ }^{11}$

While the terms super- and submodularity have been employed in economics at least since Topkis (1979), the benefit of these restrictions for combinatorial problems has not been explicitly discussed in previous work in economics. However, for many of the return functions in the literature this restriction turns out to hold, intentionally or as a side product of key economic assumptions. ${ }^{12}$

Jia (2008) models the location decisions of a chain store and derives a return function that is supermodular in the market entry decisions at individual locations. Holmes (2011) models a similar chain store decision problem but assumes negative rather than positive complementarities between different branches making his return function submodular. Arkolakis et al. (2013) present a trade application where multinational producers choose optimal plant locations across countries. The firm problem of this paper is to maximize a submodular Pseudo-Boolean objective as we show in the appendix. Antras et al. (2014) study the intensive and extensive margin of a firm's global sourcing decisions and the resulting firm profit function turns out to be either super- or submodular in the number of inputs used depending on parametric restrictions. In the appendix we provide more details on the return functions in these references.

\subsection{Additional Restrictions in the Literature}

We now present two additional restrictions on return functions in combinatorial discrete choice problems. These additional restrictions often facilitate the efficient solution finding for return functions with the loose monotonic difference property and can help to establish whether a given return function exhibits this property.

\footnotetext{
${ }^{11}$ While supermodularity and submodularity are sufficient and well-known in the economics literature, there is another weaker sufficient condition that of quasi-supermodularity and quasi-submodularity. Superand submodularity have the most intuitive economic interpretation and so we choose to discuss them in more detail.

${ }^{12}$ This is not surprising in a way given how central the ideas of complementarities, positive and negative, are in economics.
} 


\subsubsection{Analytical Return Functions}

An obvious restriction that is often imposed on combinatorial problems in economics is that of an analytical return function. The return function in Jia (2008) constitutes a particularly simple example. Since we will eventually compare our approach with the one of Jia (2008), we here state the return function of the chain store in her paper:

$$
\Pi(I)=\sum_{i=1}^{n} \underbrace{I_{i} \times\left(X_{i}+\delta \times \sum_{i^{\prime} \neq i}^{n} \frac{I_{i^{\prime}}}{\tau_{i i^{\prime}}}\right)}_{\pi_{i}(I)}
$$

Here $i$ corresponds to a particular potential store location. $I_{i}$ takes the value 1 if a store is open in location $i$ and 0 if not. In other words $I_{i}$ is a Boolean variable. $X_{i}$ is the part of the payoff from opening a store in location $i$ that is independent of other stores, which can be negative or positive. The term $\delta \times \sum I_{i^{\prime}} / \tau_{i i^{\prime}}$ in the return function parameterizes a spillover between different chain store locations, with $\tau_{i i^{\prime}}$ being the distance in miles between store location $i$ and $i^{\prime}{ }^{13}$ Jia (2008) imposes $\delta>0$ to model positive spillovers between branches, as a result (2) exhibits supermodularity.

Besides an intuitive interpretation analytical return functions have two major advantages. First, the super- or submodularity is often directly linked to a parameter in the profit function, eg. in (2) the sign of $\delta$ determines the direction of complementarities. ${ }^{14}$ This allows for easy verification of the loose increasing or decreasing differences of the objective of the problem and hence the applicability of the methods we propose. It also means estimation of the direction of complementarities translates directly into this parameter. One of the advantages of our method is that it can deal in an analogous fashion with both directions of spillovers, which means it can be conveniently nested in an estimation routine that searches over a negative and positive support for $\delta$ to minimize a loss function. The second advantage of analytical return functions is a computational one; they often allow for analytical computation of the marginal value of an index $i$, which as we will demonstrate halves the computational time of the solution method. ${ }^{15}$ Arkolakis et al. (2013) and Antras et al. (2014) are other examples from the literature that feature analytical return functions.

\footnotetext{
${ }^{13}$ Note that it is this spillover term that induces the curse of dimensionality: if there was no interaction between locations the chain store could consider all $N$ locations separately.

${ }^{14} \delta<0$ would make the return function in Jia (2008) exhibit submodularity.

${ }^{15}$ The method involves the computation of $D_{i} \Pi(I)=\Pi\left(I^{i \rightarrow 1}\right)-\Pi\left(I^{i \rightarrow 0}\right)$ for all coordinates $\mathrm{i}$ at each iteration which involves two evaluations of the profit. An analytical expression for $\Pi$ implies an analytical expression for $D_{i} \Pi(I)$ which halves the number of functions to be evaluated at each iteration.
} 


\subsubsection{Additively Separable Return Function}

Another common restriction on return functions, $\Pi$, is their separability into decision-specific return functions $\pi_{i}(I)$. These are cases in which we can rewrite (1) as follows:

$$
\Pi(I)=\sum_{i=1}^{n} \pi_{i}(I)
$$

In this case we do not restrict the functional form of the individual decision payoff function $\pi_{i}(I)$ as a function of the strategy vector $I$. However, the way individual decision payoffs are aggregated into the overall return of the agent $\Pi(I)$ is additive.

The following lemma derived directly from Costinot (2009) shows that it is enough to check for super- or submodularity in the individual return functions to guarantee that the overall return function has the respective property.

Lemma 1. If two pseudo-Boolean functions $f, g: \mathcal{B}^{n} \longrightarrow \mathbb{R}$ are supermodular then $f+g$ is supermodular. This additivity property also holds for submodular functions.

Proof. See Appendix.

The proof of this lemma follows directly from definition 2.2 and is formally stated in the appendix. Using this lemma facilitates proving that the return function $\Pi$ exhibits the super- or submodularity property and exhibits loose monotonic differences.

An array of papers feature such separable combinatorial discrete choice problems. Holmes (2011) and Jia (2008) both feature overall profit function of the chain store that takes the form 3 where $I$ is the choice of branch locations and $\pi_{i}(I)$ is the profit of the branch in location $i$. Arkolakis et al. (2013) and Tintelnot (2016) are examples from the trade literature.

The canonical Simple Plant Location Problems (SPLP) that have been studied in operation research, engineering and economics also feature separable return functions (see, for example, Balinski (1964); Manne (1964) who first formulated the problem, or Verter (2011) for a recent survey on this and related facility location problems). In these problems, given a set $n$ of potential plant locations and a finite set of demand points a chain store needs to choose the optimal distribution of stores across localities. For any location $i=1, \ldots, n$ there are fixed costs of opening a production facility and a variable cost of serving a client in another location. While the typical SPLP problem is formulated as a minimization problem it can be rewritten as a pseudo-Boolean maximization problem whose objective function takes the form of 3 (see Appendix). Using lemma 1 helps to establish the submodularity of this class of problems. 


\section{Theory}

We proceed with developing the main results of our theory. We start by defining some additional concepts in the first subsection, state our main results in the second one and finally compare our technique to existing ones.

\subsection{Definitions and Notation}

In order to state our core results we need to define some key terms from the mathematical branch of lattice theory. Lattice theory is an outgrowth of the field of Boolean algebra, and provides a framework for unifying the study of ordered sets in mathematics. Canonical references are Grätzer (2002) and Birkhoff et al. (1948).

Let $\mathbf{X}$ be a partially ordered set along with an order relation $\geq$ that is reflexive, antisymmetric and transitive. ${ }^{16}$ The partially ordered set $\mathbf{X}$ is called a lattice if it contains the least upper bound and the greatest lower bound of each pair of its elements. If $\mathbf{T}$ is a subset of a lattice $\mathbf{X}$ and $\mathbf{T}$ contains the least upper and greatest lower bound (with respect to $\mathbf{X}$ ) of each pair of elements of $\mathbf{T}$, then $\mathbf{T}$ is a sublattice of $\mathbf{X}$. A lattice $\mathbf{X}$ is complete when every nonempty subset of it has a least upper bound and a greatest lower bound contained in $\mathbf{X}$.

An important result is that the set of all permutations of the $n$-dimensional Boolean vector $I \in \mathcal{B}^{n}$ along with coordinate-wise comparison as order relation forms a complete lattice. We denote this lattice by $\mathbf{I}$ throughout the rest of the paper. We state all our results for this particular lattice, although they hold for more general lattices. Also we denote the set of all complete sublattices of the lattice $\mathbf{I}$ by $S(\mathbf{I})$ and order these sublattices by set inclusion, denote $\subseteq .^{17}$

Example 1. The two dimensional Boolean lattice is the following partially ordered set along with "coordinate-wise" comparison as an order relation:

$$
\mathbf{I}=\{(1,1),(1,0),(0,1),(0,0)\}
$$

The set is only partially ordered since under "coordinate-wise" comparison not all elements can be ranked, eg. $(0,1)$ and $(1,0)$ cannot be compared. The following lattices $\mathbf{I}^{\prime}, \mathbf{I}^{\prime \prime} \in S(\mathbf{I})$ are examples of sublattices of $\mathbf{I}$ :

$$
\mathbf{I}^{\prime}=\{(1,1),(1,0)\} \quad \mathbf{I}^{\prime \prime}=\{(0,1),(0,0)\}
$$

\footnotetext{
${ }^{16}$ Note that in the following definition an order relation is called to be reflexive if $x \geq x$ for all $x \in \mathbf{X}$, and antisymmetric if $x \geq y$ and $x \leq y$ implies that $x=y$.

${ }^{17}$ Note that the set of sublattices of $\mathbf{I}$ along with the "set-inclusion" order relation forms a complete lattice in itself.
} 
Using the "set-inclusion" order relation we see that $\mathbf{I}^{\prime} \subseteq \mathbf{I}$ while we cannot order $\mathbf{I}^{\prime}$ and $\mathbf{I}^{\prime}$, showing the sense in which the set of sublattices of $\mathbf{I}, S(\mathbf{I})$, is again a partially ordered set. Further it can be shown that $S(\mathbf{I})$ forms a complete lattice in itself.

We can now formulate the algorithm we propose as a recursive mapping which we dub as $A E: S(\mathbf{I}) \rightarrow S(\mathbf{I})$. AE maps the set of complete sublattices of $\mathbf{I}$ into itself. We say that $A E$ is set increasing if $A E(\mathbf{I}) \subseteq A E\left(\mathbf{I}^{\prime}\right)$ whenever $S(\mathbf{I}) \subseteq S\left(\mathbf{I}^{\prime}\right)$.

Definition. Consider a complete lattice $\mathbf{I}^{0}$ and form:

$$
\bar{\Omega}_{1}\left(\mathbf{I}^{0}\right)=\left\{i: D_{i}\left(\Pi\left(\sup \mathbf{I}^{0}\right)\right)<0\right\} \quad \text { and } \quad \underline{\Omega}_{1}\left(\mathbf{I}^{0}\right)=\left\{i: D_{i}\left(\Pi\left(\inf \mathbf{I}^{0}\right)\right) \geq 0\right\}
$$

Then, we define the mapping $A E_{1}: S\left(\mathbf{I}^{0}\right) \rightarrow S\left(\mathbf{I}^{0}\right)$ as

$$
A E_{1}\left(\mathbf{I}^{0}\right)=\left\{I \in \mathbf{I}^{0}: I_{i}=0 \text { and } I_{j}=1, \forall i \in \bar{\Omega}_{1}\left(\mathbf{I}^{0}\right), \forall j \in \underline{\Omega}_{1}\left(\mathbf{I}^{0}\right)\right\} .
$$

Similarly, form the following:

$$
\bar{\Omega}_{2}\left(\mathbf{I}^{0}\right)=\left\{i: D_{i}\left(\Pi\left(\sup \mathbf{I}^{0}\right)\right)>0\right\} \quad \text { and } \quad \underline{\Omega}_{2}\left(\mathbf{I}^{0}\right)=\left\{i: D_{i}\left(\Pi\left(\inf \mathbf{I}^{0}\right)\right) \leq 0\right\}
$$

Then, we define the mapping $A E_{2}: S\left(\mathbf{I}^{0}\right) \rightarrow S\left(\mathbf{I}^{0}\right)$ as

$$
A E_{2}\left(\mathbf{I}^{0}\right)=\left\{I \in \mathbf{I}^{0}: I_{i}=1 \text { and } I_{j}=0, \forall i \in \bar{\Omega}_{2}\left(\mathbf{I}^{0}\right), \forall j \in \underline{\Omega}_{2}\left(\mathbf{I}^{0}\right)\right\} .
$$

Iterating on the first mapping will help maximize objective functions that exhibit loose increasing differences, while iterating on the second will have the analogous effect for objectives exhibiting loose decreasing differences. Note that applying these mappings to a given lattice $\mathbf{I}$ corresponds to removing certain vectors from this set. Denote the lattice with the respective vectors removed after the $k$ th iteration by $\mathbf{I}^{k}$, ie. $\mathbf{I}^{k} \equiv A E_{i}\left(\mathbf{I}^{k-1}\right)$ for $i=1,2 .{ }^{18}$ Note that each $\mathbf{I}^{k}$ along with the coordinate-wise comparison order relation forms again a complete lattice. We define the complete lattice $\mathbf{I}^{k}$ such that $\mathbf{I}^{k}=A E_{i}\left(\mathbf{I}^{k}\right)$ as $\mathbf{I}^{\star}$.

\subsection{Main Results}

Our first main result is that iterating the mappings defined above on a Boolean lattice will converge to a complete lattice that contains the maximizing vector $I^{\star}=\operatorname{argmax}_{I \in \mathcal{B}^{n}} \Pi(I)$ under the sole condition that $\Pi$ exhibits the loose monotonic differences property.

Theorem 1. Consider a function $\Pi: \mathcal{B}^{n} \longrightarrow \mathbb{R}$.

\footnotetext{
${ }^{18}$ Note that $\mathbf{I}^{k}$ is a complete sublattices of $\mathbf{I}$ for any $k$.
} 
(i) If $\Pi$ exhibits loose increasing differences, the $A E_{1}: S(\boldsymbol{I}) \rightarrow S(\boldsymbol{I})$ mapping has a fixed point $\boldsymbol{I}^{\star}$ that is a complete lattice, with $I^{\star} \in \boldsymbol{I}^{\star}$.

(ii) If $\Pi$ exhibits loose decreasing differences, the $A E_{2}: S(\boldsymbol{I}) \rightarrow S(\boldsymbol{I})$ mapping has a fixed point $\boldsymbol{I}^{\star}$ that is a complete lattice, with $I^{\star} \in \boldsymbol{I}^{\star}$.

(iii) If $\Pi$ exhibits loose increasing or decreasing differences iterating on $A E_{1}$ or $A E_{2}$ identifies $\boldsymbol{I}^{\star}$ in a maximum of $n$ iterations.

Proof. See Appendix.

The loose increasing difference condition ensures that the $A E_{1}$ mapping is set increasing on the set of sublattices of $\mathbf{I}, S(\mathbf{I})$. This allows the application of the Knaster-Tarski fixed point theorem which guarantees the existence of a set of fixed points that form a complete lattice. We then show that $A E_{1}$ never eliminates $I^{\star}$ by construction, and that iterating on $A E_{1}$ always converges to the fixed point $\mathbf{I}^{\star}$. It follows that $I^{\star} \in \mathbf{I}^{\star}$. Likewise the loose decreasing difference condition makes $A E_{2}$ set-increasing on the set of sublattices of $\mathbf{I}$. We illustrate the basic workings of the $A E_{1}$ mapping in the following example.

Example 2. Let $\mathbf{I}^{0}=\{(0,0),(0,1),(1,0),(1,1)\}$. Suppose the function $\Pi$ takes the following values on $\mathbf{I}^{0}$ :

$$
\Pi(0,0)=0 \quad \Pi(0,1)=9 \quad \Pi(1,0)=-2 \quad \Pi(1,1)=11
$$

We can then easily verify that $\Pi$ exhibits loose increasing differences in this example. Indeed we can make the even stronger statement of $\Pi$ being supermodular on $\mathbf{I}^{0}$. Also note that:

$$
\sup \mathbf{I}^{0}=(1,1) \quad \inf \mathbf{I}^{0}=(0,0)
$$

Since $\Pi$ is supermodular we can apply the $A E$ algorithm. Applying $A E_{1}$ to $\mathbf{I}^{0}$ we obtain:

$D_{1}\left(\Pi\left(\sup \mathbf{I}^{0}\right)\right)=2>0 \quad D_{2}\left(\Pi\left(\sup \mathbf{I}^{0}\right)\right)=13>0 \quad D_{1}\left(\Pi\left(\inf \mathbf{I}^{0}\right)\right)=-2<0 \quad D_{2}\left(\Pi\left(\inf \mathbf{I}^{0}\right)\right)=9>0$

But then $A E_{1}\left(\mathbf{I}^{0}\right)=\mathbf{I}^{1}$ where

$$
\mathbf{I}^{1}=\{(0,1),(1,1)\}
$$

which is a complete lattice again. The application of the algorithm has effectively fixed the second entry to 1 . Applying $A E_{1}$ again, now to $\mathbf{I}^{1}$ we obtain

$$
D_{1}\left(\Pi\left(\sup \mathbf{I}^{1}\right)\right)=2>0 \quad D_{1}\left(\Pi\left(\inf \mathbf{I}^{1}\right)\right)=2>0
$$


But then $A E_{1}\left(\mathbf{I}^{1}\right)=\mathbf{I}^{2}$ where

$$
\mathbf{I}^{2}=\{(1,1)\}
$$

In this case we can find the set of fixed points of $A E_{1}$ in two steps. Further the fixed point set is a singleton. It follows that $I^{\star}=(1,1)$.

Theorem 1 is an important first step towards identifying $I^{*}$. However, at times $\mathbf{I}^{\star}$ may not be a singleton and in the worst case still contain $2^{n}$ elements. In these instances further work needs to be done to identify $I^{\star}$. For this purpose we develop an extension of the algorithm, which applies $A E$ repeatedly (and which we dub $A E-R$ (epeated) or simply $A E R)$ and which always delivers the maximizing vector $I^{*}$. We define the $A E R$ procedure as follows:

Definition. Consider a function $\Pi: \mathcal{B}^{n} \longrightarrow \mathbb{R}$ on a non-singleton complete lattice $\mathbf{I}$ that exhibits the loose increasing differences property:

(i) Iterate on $A E_{1}$ until convergence. If $\left|\mathbf{I}^{\star}\right|=1, I^{\star}=\mathbf{I}^{\star}$, else continue.

(ii) Pick any sublattices $\mathbf{I}_{1}, \mathbf{I}_{2}$ of $\mathbf{I}$, st. $\mathbf{I}=\mathbf{I}_{1} \cup \mathbf{I}_{2}$ and $\emptyset=\mathbf{I}_{1} \cap \mathbf{I}_{2}$.

(iii) Iterate $A E_{1}$ on $\mathbf{I}_{1}, \mathbf{I}_{2}$ separately resulting in fixed points $\mathbf{I}_{1}^{\star}$ and $\mathbf{I}_{2}^{\star}$.

(iv) If $\left|\mathbf{I}_{1}^{\star}\right|=\left|\mathbf{I}_{2}^{\star}\right|=1$ then $I^{\star}=\arg \max _{I \in \mathbf{I}_{1}^{\star} \cup \mathbf{I}_{2}^{\star}} \Pi(I)$.

(v) Else pick sublattices $\mathbf{I}_{i, 1}, \mathbf{I}_{i, 2}$ of $\mathbf{I}_{i}^{\star}$ st. $\mathbf{I}_{i}=\mathbf{I}_{i, 1} \cup \mathbf{I}_{i, 2}$ and $\emptyset=\mathbf{I}_{i, 1} \cap \mathbf{I}_{i, 2}$ for $i=1,2$ and repeat.

$A E R$ is defined analogously for objectives that exhibit loose decreasing differences.

$A E R$ is effectively splitting the lattice at one dimension at a time and then applies the $A E$ algorithm to the two resulting sublattices. We can prove the following theorem on the properties of the $A E R$ algorithm:

Theorem 2. If $\Pi$ exhibits the loose monotonic differences property, then AER always converges to $I^{*}$.

Proof. See Appendix.

Note that in the worst case $A E R$ collapses to the brute force approach. In terms of computational time the benefits of applying $A E R$ rather than brute force can be enormous, since $A E R$ can be thought of as yielding to brute force one coordinate at a time: whenever $A E$ cannot make progress the remaining lattice is split, implying we will need to manually compare the outcomes from running $A E$ on the two resulting sublattices. If $A E$ does not fix entries then this results in splitting an $n$ dimensional lattices $n$ times and comparing 
manually at each break point: this corresponds to the brute force approach. In practice applying $A E R$ outperformed brute force vastly in all applications.

Finally we provide a lower bound on the time complexity of the $A E$ technique:

Proposition 1. Consider a function $\Pi: \mathcal{B}^{n} \longrightarrow \mathbb{R}$ :

(i) If $\Pi$ exhibits the loose increasing difference property then $A E_{1}$ converges to $\boldsymbol{I}^{\star}$ in $O\left(n^{2}\right)$ time.

(ii) If $\Pi$ exhibits the loose decreasing difference property then $A E_{2}$ converges to $\boldsymbol{I}^{\star}$ in $O\left(n^{2}\right)$ time.

Proof. See Appendix.

The $A E R$ algorithm can be coded up as a simple recursive routine. Matlab codes of the $A E R$ method that interface with arbitrary profit functions of the form $\Pi: \mathcal{B}^{n} \longrightarrow \mathbb{R}$ are available from the authors website.

\subsection{Relation with Jia (2008)}

In order to solve the problem of maximizing the chain store return function (2), Jia (2008) develops a method to reduce the dimensionality of the problem. In this section we show how our approach nests and extends the technique outlined in Jia (2008).

Jia (2008) defines the following recursive mapping $V: \mathcal{B}^{n} \rightarrow \mathcal{B}^{n}$ :

$$
V_{i}(I):=\left[D_{i}(\Pi(I)) \geq 0\right]
$$

where $\mathbb{1}$ is an indicator function that is 1 if the argument is true. The particular contribution of Jia (2008) is that by using Theorem 1 in Tarski (1955) she shows that if $V$ is increasing on the lattice $\mathbf{I}$ (4) iterative mapping converges to a fixed point $I \in \mathcal{B}^{n}$. Her method then consists of iterating on $V$, first starting with the vector of all 1 s and then with the vector of all 0s. This way she can identify the upper and lower bound of the set of fixed points of $V$. These bounds and the set of vectors in-between them form a complete lattice $\mathbf{I}^{\star}$ that contains $I^{\star}$. Jia (2008) then evaluates $\Pi$ for all $I \in \mathbf{I}^{\star}$ to identify $I^{\star}$.

It turns out that the loose increasing differences condition of $\Pi$ is crucial for the characterization of the properties of the mapping $V$. In particular, we can prove the following lemma:

Lemma 2. $V$ is increasing if and only if $\Pi$ exhibits the loose increasing differences property.

Proof. See Appendix 
This lemma highlights that the applicability of the Jia (2008) technique is limited to return functions that exhibit the loose increasing differences property. For such return function we can prove the following lemma:

Lemma 3. If $\Pi$ exhibits the loose increasing differences property, the set of fixed points of $V$ coincides with the fixed point of $A E_{1}$.

Proof. See Appendix.

Note that $V$ is defined on the Boolean lattice, that is it takes in individual vectors and hence its fixed points are vectors. $A E_{1}$ however is defined on the set of sublattices of the Boolean lattice and operates on lattices so its fixed point is a lattice. Lemma (3) establishes that the unique fixed point of $A E_{1}$ which is a Boolean lattice is the same Boolean lattice formed by the set of fixed point vectors of the $V$ mapping. This highlights the second limitation of the Jia (2008) technique: while it can reduce the set of potential solutions in many instances there is no guarantee that the resulting set of fixed points is a singleton. In this sense the $A E R$ technique will become useful even for maximizing objectives whose set of potential solutions could previously be reduced using the Jia (2008) method. In addition the $A E$ mappings are saving on computational time, since it is not necessary to recompute marginal values for coordinates that have been fixed to 0 or 1 .

Jia (2008) also discusses the use of her algorithm in helping to identify the pure strategy Nash equilibria of a supermodular game on a lattice. In the next section we generalize these results to games that exhibit the loose monotonic difference property.

\section{Loose Monotonic Games on a Lattice}

The concept of supermodularity has gained much of its prominence in economics through its role in the theory of supermodular games. The seminal contributions to this literature are Topkis (1978) and Milgrom and Shannon (1994) who provided necessary and sufficient conditions for the solution set of a maximization problem to be monotonic in the parameters of the problem. Also Topkis (1979) and Zhou (1994) proved that a two player game with payoff functions that are supermodular in the own strategy space and have decreasing differences in the joint strategy space of the players always has a non-empty set of equilibrium points that form a complete lattice.

To our knowledge there has been no formal treatment of "submodular games", that is games in which the payoff function of one player is subdmodular in her own strategies and retains decreasing differences in the joint strategy space of the players. Jia (2008) studies a classic supermodular game in which two chain stores compete across a set of locations 
with their only choice being whether to enter a given market or not, while there are positive complementarities within each firm. A natural question to ask is then what if, as for example in the Holmes (2011) paper, there is a negative spillover effect within firms. Incorporating this feature into a market entry game across locations would lead to what we here term a "submodular game", that is a game where the profit function of an agent is submodular in his own strategy and decreasing in the joint strategy space.

We will here provide an existence proof for pure strategy Nash equilibria in $N$ player games on a lattice, where interactions are aggregative and players' profit functions are un-

restricted own actions. Note that this nests a class of super- and submodular games on a lattice for example the game considered in Jia (2008). The $A E R$ algorithm can be used to locate a Pure Strategy Nash equilibrium in such games as long as the profit function exhibits loose monotonic differences in a player's own action.

A theory of submodular games is not possible with the same generality and elegance achieved by Topkis (1979), Milgrom and Shannon (1994) and Zhou (1994) and so in this paper we restrain ourselves to a restricted class of such games.

\subsection{Setup}

Consider an $m$ player $n$ market entry game defined on the joint Boolean strategy space $\mathcal{B}^{n} \times \ldots \times \mathcal{B}^{n}$. We write player $i$ s profit function as a function of her and her opponents' strategy vectors as follows:

$$
\Pi_{i}\left(I^{1}, \ldots, I^{i}, \ldots, I^{m}\right)=f_{i}\left(I^{i}\right)+g\left(I^{1}, \ldots, I^{i}, \ldots, I^{m}\right)
$$

where $f_{i}$ is any function on $\mathcal{B}^{n}$ and allowed to differ across players and $g$ is any function on $m$ tuples of a state space $\mathbf{I}$. Note the generality of this formulation: the sole restriction on player $i$ 's profit function is that it is additively separable into a player $i$ specific part that is only a function of player $i$ 's actions, $f_{i}$, and a part that is common across players and allowed to be a function of all players' actions, $g$. This is a special case of the class of best-response potential games introduced in Voorneveld (2000) .

In particular then $f_{i}: \mathcal{B}^{n} \rightarrow \mathbb{R}$ could be a function that exhibits loose increasing or decreasing differences, or as a special case thereof super- or submodularity. In the next section we first present the result regarding the existence of pure strategy Nash equilibria for the above class of games which is a corollary of results from Voorneveld (2000). Secondly we prove that $A E R$ can always be used to identify a pure strategy Nash equilibrium in such games as long as players' profit functions $\Pi_{i}$ exhibit loose monotonic differences. 


\subsection{Using $A E R$ to Locate Equilibrium Points}

The following theorem states the condition for the existence of a pure strategy equilibrium in loose monotonic games of the form in equation (5) and gives the conditions necessary for $A E R$ to be useful for locating such equilibrium points, if they exist.

Theorem 3. Consider the $n$-player m-market game in equation (5). In such games

(i) The set of pure strategy Nash equilibria is non-empty.

(ii) If $\Pi_{i}$ exhibits the loose monotonic difference condition for all $i$, iteratively applying AER for all players eventually terminates in a pure strategy Nash equilibrium.

Proof. See Appendix.

Part $(i)$ is a corollary of results in Voorneveld (2000). The proof is based on showing that there exist no best response cycles. One can start with any player $i$ and solve her problem holding the actions of all other players fixed, then fix $i$ 's strategy and move on to the next player solving her problem holding the actions of all players including $i$ fixed. Voorneveld (2000) proved that in games of the form above such a procedure exhibits no cycles and hence, since there is a finite amount of paths through any players' finite action space, there must be at least one pure strategy Nash equilibrium. $A E R$ can solve the individual player's problem at each point in this process if the $\Pi_{i}$ exhibits loose monotonic differences and hence will always identify a Nash equilibrium in such games.

\subsection{Special Cases}

In this section we show some special cases of the general formulation of loose monotonic difference games in equation (5). In the first subsection we demonstrate how it nests the game in Jia (2008), while in the second we outline a two-player version which makes the game non-aggregative.

\subsubsection{Quadratic Games}

We note that a special case of the class of games defined above are quadratic super- or submodular games of the following form, where the sign of $\delta_{i, j}$ determines the direction of complementarities, while the sign of $c_{j}$ determines whether $\Pi_{i}$ is increasing or decreasing in the joint strategy space.

$$
\Pi_{i}\left(I^{1}, \ldots, I^{i}, \ldots, I^{m}\right)=\sum_{j=1}^{n}\left(\mu_{j} I_{j}^{i}+\sum_{k \neq j} \delta_{i, j} I_{j}^{i} I_{k}^{i}-\sum_{l \neq i} c_{j} I_{j}^{i} I_{j}^{l}\right)
$$


In the case with just two players, $\delta_{i, j}$ and $c_{j}$ restricted to be positive, this is the game considered in Jia (2008).

\subsubsection{Two player Games}

In the two player games we can write the game in the following general form:

$$
\begin{aligned}
& \Pi_{1}\left(I^{1}, I^{2}\right)=f_{1}\left(I^{1}\right)+g\left(I^{1}, I^{2}\right) \\
& \Pi_{2}\left(I^{1}, I^{2}\right)=f_{2}\left(I^{2}\right)+g\left(I^{1}, I^{2}\right)
\end{aligned}
$$

In this special case the aggregate of all players' actions $g\left(I^{1}, I^{2}\right)$ is just a function of the opponent and hence the game is now more general than aggregative games.

\section{Conclusion}

This paper introduces a class of combinatorial discrete choice problems along with a method to solve them. We introduced intuitive and easy to implement procedures that can be used to simplify and solve optimization problems in the class of combinatorial discrete choice problems characterized by the loose monotonic differences property. Further we described a class of multiplayer games on multidimensional Boolean lattices in which the existence of a pure strategy Nash equilibrium is guaranteed and an equilibrium point can always be computed. 


\section{References}

Acemoglu, D. And M. K. Jensen (2013): "Aggregate comparative statics," Games and Economic Behavior, 81, 27-49.

Antràs, P. And A. De Gortari (2017): "On the Geography of Global Value Chains," Harvard mimeo.

Antras, P., T. C. Fort, And F. Tintelnot (2014): "The margins of global sourcing: theory and evidence from US firms," Tech. rep., National Bureau of Economic Research.

Arkolakis, C., N. Ramondo, A. Rodríguez-Clare, and S. Yeaple (2013): "Innovation and production in the global economy," Tech. rep., National Bureau of Economic Research.

BALINSKI, M. (1964): "On finding integer solutions to linear programs," Tech. rep., DTIC Document.

BALINSKi, M. L. (1965): "Integer programming: methods, uses, computations," Management Science, 12, 253-313.

Birkhoff, G., G. Birkhoff, G. Birkhoff, E.-U. Mathématicien, and G. Birkhoff (1948): Lattice theory, vol. 25, American Mathematical Society New York.

Boros, E. And P. L. Hammer (2002): "Pseudo-boolean optimization," Discrete applied mathematics, 123, 155-225.

Carvalho, V. M. and N. Voigtländer (2014): "Input diffusion and the evolution of production networks," Tech. rep., National Bureau of Economic Research.

Cornuéjols, G., G. L. Nemhauser, and L. A. Wolsey (1983): "The uncapacitated facility location problem," Tech. rep., DTIC Document.

Costinot, A. (2009): "An Elementary Theory of Comparative Advantage," Econometrica, $77,1165-1192$.

Eaton, J. And S. Kortum (2002): "Technology, geography, and trade," Econometrica, 70, $1741-1779$.

Grätzer, G. (2002): General lattice theory, Springer Science \& Business Media.

Hansen, P. And J.-F. Thisse (1977): "Multiplant location for profit maximisation," Environment and Planning A, 9, 63-73. 
Hendel, I. (1999): "Estimating multiple-discrete choice models: An application to computerization returns," The Review of Economic Studies, 66, 423-446.

Holmes, T. J. (2011): "The Diffusion of Wal-Mart and Economies of Density," Econometrica, 79, 253-302.

JiA, P. (2008): "What Happens When Wal-Mart Comes to Town: An Empirical Analysis of the Discount Retailing Industry," Econometrica, 76, 1263-1316.

Knaster, B. (1928): "Un théorème sur les fonctions d'ensembles," Ann. Soc. Polon. Math., $6,133 ? 134$.

Krarup, J. And P. M. Pruzan (1983): "The simple plant location problem: survey and synthesis," European Journal of Operational Research, 12, 36-81.

Manne, A. S. (1964): "Plant location under economies-of-scale-decentralization and computation," Management Science, 11, 213-235.

Milgrom, P. And C. Shannon (1994): "Monotone comparative statics," Econometrica: Journal of the Econometric Society, 157-180.

Morales, E., G. Sheu, And A. Zahler (2015): "Extended Gravity," .

Owen, S. H. AND M. S. DASKIn (1998): "Strategic facility location: A review," European Journal of Operational Research, 111, 423-447.

Pakes, A., J. Porter, K. Ho, And J. Ishit (2015): "Moment inequalities and their application," Econometrica, 83, 315-334.

TARski, A. (1955): "A lattice-theoretical fixpoint theorem and its applications," Pacific journal of Mathematics, 5, 285-309.

Tintelnot, F. (2016): "Global production with export platforms," The Quarterly Journal of Economics, qjw037.

Topkis, D. M. (1978): "Minimizing a submodular function on a lattice," Operations research, 26, 305-321.

(1979): "Equilibrium points in nonzero-sum n-person submodular games," Siam Journal on control and optimization, 17, 773-787.

VERTER, V. (2011): "Uncapacitated and capacitated facility location problems," in Foundations of location analysis, Springer, 25-37. 
VIVES, X. (1990): "Nash equilibrium with strategic complementarities," Journal of Mathematical Economics, 19, 305-321.

Voorneveld, M. (2000): "Best-response potential games," Economics letters, 66, 289-295.

Wolpert, D. H. and W. G. Macready (1997): "No free lunch theorems for optimization," IEEE transactions on evolutionary computation, 1, 67-82.

Zhou, L. (1994): "The set of Nash equilibria of a supermodular game is a complete lattice," Games and economic behavior, 7, 295-300. 


\section{A Appendix}

The authors want to thank Jake DiCicco, Andrew Salmon, Benjamin Tong, and Yunus Tuncbilek for their outstanding help with this Appendix.

\section{A.1 Proofs of Results}

\section{Proof of Lemma 1}

Lemma. If two pseudo-Boolean functions $f, g: \mathcal{B}^{n} \longrightarrow \mathbb{R}$ are supermodular then $f+g$ is supermodular. This additivity property also holds for submodular functions.

Proof. From the definition of supermodularity of a Pseudo-Boolean function we know that for $f$ and $g$ the following holds:

$$
\begin{aligned}
& f(\max (x, y))-f(x) \geq f(y)-f(\min (x, y)) \\
& g(\max (x, y))-g(x) \geq g(y)-g(\min (x, y))
\end{aligned}
$$

But this then implies for $f+g$ :

$$
\begin{gathered}
{[f(\max (x, y))-f(x)]+[g(\max (x, y))-g(x)] \geq[f(y)-f(\min (x, y))]+[g(y)-g(\min (x, y))]} \\
{[g+f](\max (x, y))-[g+f](x) \geq[g+f](y)-[g+f](\min (x, y))}
\end{gathered}
$$

Therefore $g+f$ is also supermodular. The proof for the submodular case works analogously.

\section{Proof of Theorem 1}

Theorem. Consider a function $\Pi: \mathcal{B}^{n} \longrightarrow \mathbb{R}$.

(i) If $\Pi$ exhibits loose increasing differences, the $A E_{1}: S(\boldsymbol{I}) \rightarrow S(\boldsymbol{I})$ mapping has a fixed point $\boldsymbol{I}^{\star}$ that is a complete lattice, with $I^{\star} \in \boldsymbol{I}^{\star}$.

(ii) If $\Pi$ exhibits loose decreasing differences, the $A E_{2}: S(\boldsymbol{I}) \rightarrow S(\boldsymbol{I})$ mapping has a fixed point $\boldsymbol{I}^{\star}$ that is a complete lattice, with $I^{\star} \in \boldsymbol{I}^{\star}$.

(iii) If $\Pi$ exhibits loose increasing or decreasing differences iterating on $A E_{1}$ or $A E_{2}$ identifies $\boldsymbol{I}^{\star}$ in a maximum of $n$ iterations. 
To proof this theorem we proceed in several steps. First define the following mapping $V: \mathcal{B}^{n} \rightarrow \mathcal{B}^{n}$ such that

$$
V_{i}(I):=\mathbb{1}\left[D_{i}(\Pi(I)) \geq 0\right]
$$

In a first step we prove that this mapping is increasing if and only if $\Pi$ exhibits loose increasing differences and decreasing if $\Pi$ exhibits loose decreasing differences.

Lemma. $V$ is increasing (decreasing) if and only if the loose increasing (decreasing) difference condition holds for $\Pi$.

Proof. First, suppose that $V$ is increasing. Consider any $1 \leq i \leq n$. Then, consider any two vectors $I, I^{\prime}$ s.t. $I^{\prime} \leq I$. If $D_{i}\left(\Pi\left(I^{\prime}\right)\right) \geq 0$, then $V_{i}\left(I^{\prime}\right)=1$, and since $V$ is increasing, $V_{i}(I)=1$, which implies that $D_{i}(\Pi(I)) \geq 0$. Since this holds for any two vectors and for any $i$, we must have that the loose increasing difference condition holds.

Next, suppose that the loose increasing difference condition holds. Consider any $1 \leq i \leq n$. Then, consider any two vectors $I, I^{\prime}$ s.t. $I^{\prime} \leq I$. If $V_{i}\left(I^{\prime}\right)=1$, then $D_{i}\left(\Pi\left(I^{\prime}\right)\right) \geq 0$, so by the loose increasing difference condition, $D_{i}(\Pi(I)) \geq 0$, so $V_{i}(I)=1$. Otherwise, if $V_{i}\left(I^{\prime}\right)=0$, then $V_{i}(I) \geq V_{i}\left(I^{\prime}\right)$ since $V$ can only take on the values 0 or 1 . Since this holds for any two vectors and for any $i$, we must have that $V$ is increasing.

An analogous proof holds for the decreasing case.

Next we show that $A E_{1}$ is set increasing if and only if $V$ is increasing and $A E_{2}$ is set increasing if and only if $V$ is decreasing.

Lemma. $A E_{1}$ is set-increasing if and only if $V$ is increasing. $A E_{2}$ is set-increasing if and only if $V$ is decreasing.

Proof. Suppose first that $V$ is increasing. Consider any two lattices $\mathbf{I}, \mathbf{I}^{\prime} \subseteq \mathcal{B}^{n}$ such that $\mathbf{I}^{\prime} \subseteq \mathbf{I}$. We want to show that $A E_{1}\left(\mathbf{I}^{\prime}\right) \subseteq A E_{1}(\mathbf{I})$. Since $\mathbf{I}^{\prime} \subseteq \mathbf{I}$, we know that

$$
\begin{gathered}
\inf \mathbf{I} \leq \inf \mathbf{I}^{\prime} \\
\sup \mathbf{I} \geq \sup \mathbf{I}^{\prime} .
\end{gathered}
$$

Then, since $V$ is increasing, we also have that for all $1 \leq i \leq n$,

$$
\begin{aligned}
D_{i}(\Pi(\inf \mathbf{I})) & \leq D_{i}\left(\Pi\left(\inf \mathbf{I}^{\prime}\right)\right) \\
D_{i}(\Pi(\sup \mathbf{I})) & \geq D_{i}\left(\Pi\left(\sup \mathbf{I}^{\prime}\right)\right) .
\end{aligned}
$$


Now suppose index $j$ was set to 1 in $A E_{1}(\mathbf{I})$. Therefore by the definition of $A E_{1}$, we know that we must have $D_{j}(\Pi(\inf \mathbf{I})) \geq 0$. But since $D_{i}(\Pi(\inf \mathbf{I})) \leq D_{i}\left(\Pi\left(\inf \mathbf{I}^{\prime}\right)\right.$ for all $i$, we also have $D_{j}\left(\Pi\left(\inf \mathbf{I}^{\prime}\right)\right) \geq 0$, which means that index $j$ is set to 1 in $A E_{1}\left(\mathbf{I}^{\prime}\right)$ as well. Similarly, if index $j$ was set to 0 in $A E_{1}(\mathbf{I})$, by the same reasoning with sup instead, we know that index $j$ was set to 0 in $A E_{1}\left(\mathbf{I}^{\prime}\right)$. Therefore, $A E_{1}\left(\mathbf{I}^{\prime}\right)$ has at least as many set indices as $A E_{1}(\mathbf{I})$, which means that $A E_{1}\left(\mathbf{I}^{\prime}\right) \subseteq A E_{1}(\mathbf{I})$, thereby showing that $A E_{1}$ is set-increasing. If $V$ is decreasing, the proof for $A E_{2}$ is analogous.

Now suppose that $A E_{1}$ is set-increasing. Assume by contradiction that $V$ is not increasing. Consider two vectors $I^{\prime}, I^{\prime \prime} \in \mathcal{B}^{n}$ such that $I_{i}^{\prime}=0, I_{i}^{\prime \prime}=1$ for some $i, I_{j}^{\prime}=I_{j}^{\prime \prime}$ for all $j \neq i$. Then there must exist an index $k$ such that $V_{k}\left(I^{\prime}\right)=1$ while $V_{k}\left(I^{\prime \prime}\right)=0$ since else $V$ would be increasing. This implies that $D_{k}\left(\Pi\left(I^{\prime}\right)\right) \geq 0$ and $D_{k}\left(\Pi\left(I^{\prime \prime}\right)\right)<0$. Note that $k \neq i$, since otherwise the two derivatives would necessarily be equal.

Let $\mathbf{I}^{\prime \prime}=\left\{I: I \leq I^{\prime \prime}\right\}$ and $\mathbf{I}^{\prime}=\left\{I: I \leq I^{\prime}\right\}$, so $\mathbf{I}^{\prime} \subseteq \mathbf{I}^{\prime \prime}$. Since $A E_{1}$ is set-increasing, $A E_{1}\left(\mathbf{I}^{\prime}\right) \subseteq A E_{1}\left(\mathbf{I}^{\prime \prime}\right)$. Since $D_{k}\left(\Pi\left(\sup \mathbf{I}^{\prime \prime}\right)\right)=D_{k}\left(\Pi\left(I^{\prime \prime}\right)\right)<0, A E_{1}\left(\mathbf{I}^{\prime \prime}\right)$ must have index $k$ set to 0 , which means that $A E_{1}\left(\mathbf{I}^{\prime}\right)$ must have index $k$ set to 0 as well since $A E_{1}$ set-increasing implies $A E_{1}\left(\mathbf{I}^{\prime}\right) \subseteq A E_{1}\left(\mathbf{I}^{\prime \prime}\right)$.

Let $\mathbf{K}^{\prime \prime}=\left\{I: I \geq I^{\prime \prime}\right\}$ and $\mathbf{K}^{\prime}=\left\{I: I \geq I^{\prime}\right\}$, so $\mathbf{K}^{\prime \prime} \subseteq \mathbf{K}^{\prime}$. Since $D_{k}\left(\Pi\left(\inf \mathbf{K}^{\prime}\right)\right)=$ $D_{k}\left(\Pi\left(I^{\prime}\right)\right) \geq 0, A E_{1}\left(\mathbf{K}^{\prime}\right)$ must have index $k$ set to 1 , which means that $A E_{1}\left(\mathbf{K}^{\prime \prime}\right)$ must have index $k$ set to 1 as well since $A E_{1}$ set-increasing implies $A E_{1}\left(\mathbf{K}^{\prime \prime}\right) \subseteq A E_{1}\left(\mathbf{K}^{\prime}\right)$.

If $I_{k}^{\prime}=I_{k}^{\prime \prime}=0$, then $\mathbf{K}^{\prime}$ must contain at least 1 vector with index $k$ set to 0 . In order for $A E_{1}\left(\mathbf{K}^{\prime \prime}\right)$ to only have vectors with index $k$ set to 1 , we must have that $D_{k}\left(\Pi\left(\inf \mathbf{K}^{\prime \prime}\right)\right)=$ $D_{k}\left(\Pi\left(I^{\prime \prime}\right)\right) \geq 0$, which is a contradiction.

Therefore, we need that $I_{k}^{\prime}=I_{k}^{\prime \prime}=1$. Then, $\mathbf{I}^{\prime}$ must contain at least 1 vector with index $k$ set to 1 . In order for $A E_{1}\left(\mathbf{I}^{\prime}\right)$ to only have vectors with index $k$ set to 0 , we must have that $D_{k}\left(\Pi\left(\sup \mathbf{I}^{\prime}\right)\right)=D_{k}\left(\Pi\left(I^{\prime}\right)\right)<0$. But this is also a contradiction.

Therefore by contradiction $V$ must be increasing. Similarly, it can be shown that $A E_{2}$ set-increasing implies that $V$ is decreasing.

Together then the last two lemmas imply that $A E_{1}$ is set increasing if and only if $\Pi$ exhibits loose increasing differences and that $A E_{2}$ is set increasing if and only if $\Pi$ exhibits loose decreasing differences. 
Theorem. If $A E_{1}$ is set increasing, then it converges to a complete lattice that includes $I^{\star}$. If $A E_{2}$ is set increasing, then it converges to a complete lattice that includes $I^{\star}$.

Proof. Consider the $A E_{1}$ mapping. By construction if $I^{\star} \in \mathbf{I}$, then $I^{\star} \in A E_{1}(\mathbf{I})$. Also note since $A E_{1}$ is set increasing and $S(\mathbf{I})$, the set of all sublattices of $\mathbf{I}$, forms a complete lattice we can invoke the Knaster Tarski theorem to guarantee that $A E_{1}$ has a non-empty set of fixed points on $S(\mathbf{I})$ that forms a complete lattice. Call that set of fixed point $S\left(\mathbf{I}^{\star}\right)$, so that $A E_{1}\left(\mathbf{I}^{\prime}\right)=\mathbf{I}^{\prime}$ for all $\mathbf{I}^{\prime} \subseteq S\left(\mathbf{I}^{\star}\right)$. But then $I^{\star} \in \mathbf{I}^{\star}$, where $\mathbf{I}^{\star}$ is the largest fixed point of $A E_{1}$. Therefore, the proof is complete.

To see why (iii) in Theorem 1 holds is immediate. Note that the $A E$ mappings fix at least one entry each iteration, since else a fixed point is reached. Since all vectors in $\mathbf{I}$ have $n$ entries, both $A E$ mappings always converge in a maximum of $n$ steps. This completes the proof.

\section{Proof of Theorem 2}

From the proof of theorem 1 we know that $A E_{1}$ is set-increasing if and only if $\Pi$ exhibits the loose increasing differences property. We also know that $A E_{2}$ is set-increasing if and only if $\Pi$ exhibits the loose decreasing property. But then to prove the statement of theorem 2 all we need to prove is that if $A E_{1}$ or $A E_{2}$ are set increasing then $A E R$ always identifies $I^{\star}$.

Theorem. If $A E_{1}$ is set increasing then $A E R$ always identifies $I^{*}$. If $A E_{2}$ is set increasing then AER always identifies $I^{*}$.

Proof. Consider an objective function $\Pi$ that exhibits loose increasing differences. We use induction on $n$, the dimension of the lattice. For the base case, if $n=1$, then it is trivial in determining $I^{*}$, as there are only two options to choose from, either $I=1$ or $I=0$. Applying $A E_{1}$, if $D_{1} \Pi(I)>0 I^{\star}=1$, else $I^{\star}=0$.

Now suppose that $A E R$ always identifies $I^{*}$ given some $I \in \mathcal{B}^{k}$. We want to show that $A E R$ always identifies $I^{*}$ given some $I$ of dimension $k+1$. Given $I \in \mathcal{B}^{k+1}$, we try to run $A E_{1}$. We have two cases.

The first case is that $A E_{1}$ fixes some $a$ indices, where $a>0$. Then, $A E_{1}(\mathbf{I}) \subset \mathbf{I}$ is a proper subset of $\mathbf{I}, \mathbf{I}^{1}=A E_{1}(\mathbf{I})$. We can consider $\mathbf{I}^{1}$ to be of dimension $k+1-a$, as $a$ dimensions are fixed. By construction, $I^{*}$ is included in $A E_{1}(\mathbf{I})$. Therefore, by the inductive hypothesis, $A E R$ will identify $I^{*}$ from $A E_{1}(\mathbf{I})$. 
The second case is that $A E$ fixes no indices. In this case, we form two sublattices of $\mathbf{I}$, $\mathbf{I}^{i \rightarrow 1}$ and $\mathbf{I}^{i \rightarrow 0}$. Since we fixed one index in both these new sublattices, $\mathbf{I}^{i \rightarrow 1}$ and $\mathbf{I}^{i \rightarrow 0}$ can be considered of dimension $k$. Furthermore, $I^{*} \in \mathbf{I}^{i \rightarrow 1}$ or $I^{*} \in \mathbf{I}^{i \rightarrow 0}$, since the $\mathbf{I}$ is the disjoint union of these two sublattices. Let $\mathbf{I}^{\star i \rightarrow 1}$ be the vector returned from applying $A E R$ to the lattice $\mathbf{I}^{i \rightarrow 1}$ and $\mathbf{I}^{ \pm i \rightarrow 0}$ be the vector returned from applying $A E R$ to the lattice $\mathbf{I}^{i \rightarrow 0}$. By the inductive hypothesis, these two vectors are the optimal vectors for their corresponding lattices. Then, by $A E R$ on $\mathbf{I}$, we return the vector among these two that product the optimal profit. But since $I^{*}$ is in one of these sublattices, we must have that $I^{*}$ is equal to $\mathbf{I}^{\star i \rightarrow 1}$ or $\mathbf{I}^{\star i \rightarrow 0}$. Thus, in this case, $A E R$ also identifies $I^{*}$.

Therefore, the inductive step is proven, and we have shown that AER always identifies $I^{*}$ for any fixed dimension $n \geq 1$. The proof for an objective function that exhibits loose decreasing differences works analogously.

\section{Proof of Proposition 1}

Proposition. Consider a function $\Pi: \mathcal{B}^{n} \longrightarrow \mathbb{R}$ :

(i) If $\Pi$ exhibits the loose increasing difference property then $A E_{1}$ converges to $\boldsymbol{I}^{\star}$ in $O\left(n^{2}\right)$ time.

(ii) If $\Pi$ exhibits the loose decreasing difference property then $A E_{2}$ converges to $\boldsymbol{I}^{\star}$ in $O\left(n^{2}\right)$ time.

Proof. Similar to the logic for the proof of part (iii) in Theorem 1. $A E_{1}$ takes a maximum of $n$ iterations to converge to its fixed point by Theorem 1.(iii). At each iteration there are a maximum of $2 \times n+2$ functions to evaluate to compute all derivatives. It follows that the algorithm has a time complexity of $\mathcal{O}\left(n^{2}\right)$.

\section{Proof of Lemma 2}

Lemma. $V$ is increasing if and only if $\Pi$ exhibits the loose increasing differences property. Proof. Lemma 2 is the first lemma in the proof of theorem 1.

\section{Proof of Lemma 3}

Lemma. If $\Pi$ exhibits the loose increasing differences property, the set of fixed points of $V$ coincides with the fixed point of $A E_{1}$.

Proof. Consider the set of fixed points of $V$ and denote it $\mathcal{A} \subseteq \mathcal{B}^{n}$. Also consider the lattice that forms the fixed point of $A E_{1}$ and denote it $\mathbf{I}^{\star} \subseteq \mathcal{B}^{n}$. 
Firstly suppose there exists a vector $I \in \mathcal{A}$ such that $I \notin \mathbf{I}^{\star}$. Since $I \in \mathcal{A}$ we know that $D_{i} \Pi(I) \geq 0 \forall i I_{i}=1$ and $D_{i} \Pi(I)<0 \forall i I_{i}=0$. Now note that $I=\sup \{I\}=\inf \{I\}$. But then $D_{i} \Pi(\inf I) \geq 0 \forall i I_{i}=1$ and $D_{i} \Pi(\sup I)<0 \forall i I_{i}=0$. Applying $A E_{1}$ to $I$ would result

in $I$, ie. $\{I\}=A E_{1}(\{I\})$. Hence $A E_{1}$ would never eliminate $I$ and so $I$ is part of the lattice that forms the fixed point of $A E_{1}$. So $I \in \mathbf{I}^{\star}$ and hence we have a contradiction. So if $I \in \mathcal{A}$ then $I \in \mathbf{I}^{\star}$.

Secondly suppose there exists a vector $I \in \mathbf{I}^{\star}$ such that $I \notin \mathcal{A}$. Now note that $I=$ $\sup \{I\}=\inf \{I\}$. Since $I \in \mathbf{I}^{\star}$ we know $D_{i} \Pi(\inf I) \geq 0 \forall i I_{i}=1$ and $D_{i} \Pi(\sup I)<0 \forall i I_{i}=$ 0 . But then $I \in \mathcal{A}$ and hence we have a contradiction. So if $I \in \mathbf{I}^{\star}$ then $I \in \mathcal{A}$. Hence $\mathbf{I}^{\star} \subseteq \mathcal{A}$ and $\mathcal{A} \subseteq \mathbf{I}^{\star}$ so $\mathcal{A}=\mathbf{I}^{\star}$ and the proof is complete.

\section{Proof of Theorem 3}

Theorem. Consider the $n$-player $m$-market game above with $\Pi_{i}($.$) exhibiting the loose$ monotonic difference condition for all $i$. In such games

(i) The set of pure strategy Nash equilibria is non-empty.

(ii) Iteratively applying AER for all players eventually terminates in a pure strategy Nash equilibrium.

Proof. For part (i) simply note that $P\left(I_{1}, \ldots, I_{i}, \ldots, I_{n}\right)=g\left(I_{1}, \ldots, I_{i}, \ldots, I_{n}\right)+\sum_{i=1}^{n} f_{i}\left(I_{i}\right)$ is a potential function in the sense that the vectors in $\mathbf{I}$ maximizing the profit function $\Pi_{i}$ for player $i$ also maximize the potential function $P$ with the actions of all other players $j \neq i$ fixed. The result follows by a result from Voorneveld (2000). The existence of the so called potential function $P$ makes the game above a best response potential game in the sense of Voorneveld (2000).

For (ii) simply note that by Voorneveld (2000) Theorem 3.2 a best response potential game exhibits no best response cycles. Also note that since $\Pi_{i}$ exhibits loose monotonic differences we know that $A E R$ can identify $I^{\star}=\arg \max \Pi_{i}$ for all $I^{j} j \neq i$ fixed. But then starting from player $i=1$ we can solve for $I^{\star}=\arg \max \Pi_{i}$ by taking the strategies of all other players as given at their current $I^{\star}$. Since there are no best response cycles and strategy sets are finite this procedure will always terminate in a pure strategy Nash equilibrium. This completes the proof. 


\section{A.2 Examples of Pseudo-Boolean Return Functions in Literature}

\section{A.2.1 Jia (2008)}

In Jia (2008) a chain store chooses an optimal set of store locations across the United States. There is a large number $n$ of potential store locations from which it has to select a subset so as to maximize its overall profits. Jia (2008) postulates positive spillovers between different branches as a result of scale economies. In particular, the overall profit function in Jia (2008) can be written:

$$
\Pi(I)=\sum_{i=1}^{n} I_{i} \times\left(X_{i}+\delta \times \sum_{i^{\prime} \neq i}^{n} \frac{I_{i^{\prime}}}{\tau_{i i^{\prime}}}\right)
$$

Here $i$ corresponds to a particular potential store location. $X_{i}$ is the part of the payoff from opening a store in location $i$ that is independent of other branches. The term $\delta \sum I_{i^{\prime}} / \tau_{i i^{\prime}}$ parameterizes the positive spillover between different branches, with $\tau_{i i^{\prime}}$ being the distance in miles between store location $i$ and $i^{\prime}$. Jia (2008) proves in Appendix $B 4$ of her paper that the function (6) is supermodular on the Boolean Lattice space. Hence the $A E R$ algorithm can be applied to the problem. The method Jia (2008) uses to reduce the problem corresponds to a single application of the AE algorithm.

\section{A.2.2 Arkolakis et al. (2013)}

Arkolakis et al. (2013) build a model of trade and multi-national production. Firms can locate production sites outside their home market and use these locations to serve both the home market and/or other international markets. The problem of an individual firm at home in country $k$ is to choose a vector $I \in \mathcal{B}^{n}$ where $n$ is the number of countries and where $I_{i}=1$ corresponds to the decision to build a plant in location $i$ at fixed cost $f_{i}$. Conditional on a given plant location vector $I$ we can then calculate the optimal quantities that a given plant in $i$ is supplying to all $n$ possible markets. Denote by $\pi_{i}(I)$ the net profit plant $i$ makes from selling to all locations for which it is the lowest cost supplier given the current plant strategy $I$. If we denote the profit of plant $i$ from serving market $l$ by $\pi_{i l}$ for which it is the lowest cost supplier we have $\pi_{i}(I)=\sum_{l} \pi_{i l}$. We denote by $\mathbf{J}(I)$ a matrix with $J_{i l}=1$ if location $i$ is the cheapest cost supplier to location $l$ (among all locations of the firm around the world for which $I_{i}=1$ ). The profit function of a firm then can be written as

$$
\Pi(I)=\sum_{i=1}^{n} \mathbb{1}\left[I_{i}=1\right]\left(\sum_{l=1}^{n} \mathbb{1}\left[J_{i l}(I)=1\right] \pi_{i l}-w_{i} f_{i}\right)
$$


where

$$
\pi_{k l}=\left\{\begin{array}{lll}
\frac{1}{\sigma}\left(\frac{\sigma}{\sigma-1}\right)^{1-\sigma}\left(C_{i k l}\right)^{1-\sigma} X_{l} P_{l}^{\sigma-1}-f_{l} w_{l} & \text { if } & J_{k l}=1 \\
0 & \text { if } \quad J_{k l}=0
\end{array}\right.
$$

$\sigma$ is the elasticity of substitution between varieties, $C_{k i l}$ is the unit cost of a firm from $k$ producing in location $i$ to serve market $l$. There is a fixed cost of entering market $l$ which is paid in country $l$ wages and $X_{l} P_{l}^{1-\sigma}$ is a measure of local demand in destination $l$. Suppose plant $i$ makes a profit of $\pi_{i}(I)$ intially and then other plants are opened up. Plant $i$ 's profit will weakly decrease in the presence of the additional plants since it may cease to be the cheapest supplier for some destinations. Hence the function (7) is submodular on the Boolean lattice and $A E R$ can be applied to identify $I^{\star}$.

\section{A.2.3 Holmes (2011)}

In Holmes (2011) a chain store chooses in which locations across the United States to open an outlet. In contrast to Jia (2008), Holmes (2011) assumes that different stores are substitutes for consumers so that there is business stealing between different store locations. The revenue of a given store in location $i$ is given by $r_{i}(I)$. Variable profit of location $i$ can be written as $\bar{\nu} r_{i}(\mathbf{I})$, where $\bar{\nu}$ is taken from the data. There is also a location specific fixed cost $f_{i}$. The overall chain store profit function can be written:

$$
\Pi(I)=\sum_{i=1}^{n} \mathbb{1}\left[I_{i}=1\right]\left(\bar{\nu} r_{i}(I)-f_{i}\right)
$$

The business stealing effect implies that the function (8) is submodular on a lattice and $A E R$ can be applied to identify $I^{\star}$.

\section{A.2.4 Antras et al. (2014)}

Antras et al. (2014) study the intensive and extensive margin of a firm's global sourcing decision. The extensive margin decision can be cast as a combinatorial problem: an individual firm in country $k$ needs to decide from which countries $i=1, \ldots, n$ to source inputs. Sourcing from $i$ incurs a fixed cost $w_{i} f_{i}$. Conditional on sourcing from $i$ the problem is an Eaton and Kortum (2002) type intensive margin problem. We can then first solve the intensive margin problem conditional on buying from $i$, and then rephrase the problem as a Boolean one that fits our setup. The firm profit function can be written: 


$$
\Pi(I)=\phi^{\sigma-1}\left(\gamma \sum_{i=1}^{n} \mathbb{1}\left[I_{i}=1\right] \frac{T_{i}}{\left(\tau_{k i} w_{i}\right)^{\theta}}\right)^{\frac{\sigma-1}{\theta}} B_{k}-w_{k} \sum_{i=1}^{n} \mathbb{1}\left[I_{i}=1\right]\left(w_{i} f_{i}\right)
$$

$\phi$ is a firm specific productivity and $B_{k}$ is a measure of market demand in the home market $k$. $\gamma$ is some constant, $T_{i}$ is the mean of the source $i$ Frechet productivity distribution, $\theta$ its dispersion parameter, $\tau_{k i}$ iceberg trade costs between countries $k$ and $i$ and $w_{i}$ the wage in country $i$. As above $\sigma$ is the elasticity of substitution across varieties. As the authors show in the paper, when $\frac{\sigma-1}{\theta}>1$ the profit function is supermodular. This is the assumption the authors make in order to be able apply the Jia (2008) algorithm. Additionally, when $\frac{\sigma-1}{\theta}<1$, the opposite statements are true and the profit function is submodular. In either cases the vector $I^{\star}$ that maximizes (9) can be found by applying $A E R$.

\section{A.3 Additional Results and Counterexamples}

\section{A.3.1 An alternative sufficient condition for loose monotonic differences}

Definition. A function $f: \mathcal{B}^{n} \rightarrow \mathbb{R}$ is quasi-supermodular if the following two conditions are satisfied:

$$
\begin{aligned}
& f(I) \geq f\left(\min \left(I, I^{\prime}\right)\right) \Longrightarrow f\left(\max \left(I, I^{\prime}\right)\right) \geq f\left(I^{\prime}\right) \\
& f(I)>f\left(\min \left(I, I^{\prime}\right)\right) \Longrightarrow f\left(\max \left(I, I^{\prime}\right)\right)>f\left(I^{\prime}\right) .
\end{aligned}
$$

A function $f: \mathcal{B}^{n} \rightarrow \mathbb{R}$ is quasi-submodular if the following two conditions are satisfied:

$$
\begin{aligned}
f(I) \leq f\left(\min \left(I, I^{\prime}\right)\right) & \Longrightarrow f\left(\max \left(I, I^{\prime}\right)\right) \leq f\left(I^{\prime}\right) \\
f(I)<f\left(\min \left(I, I^{\prime}\right)\right) & \Longrightarrow f\left(\max \left(I, I^{\prime}\right)\right)<f\left(I^{\prime}\right) .
\end{aligned}
$$

The next proposition shows that quasi-supermodularity is a weaker concept than supermodularity. The same is true for quasi-submodularity and submodularity.

Proposition 2. If $f$ is a supermodular function, then $f$ is also quasisupermodular. If $f$ is a submodular function, then $f$ is also quasi-submodular.

Proof. Suppose $f$ is supermodular. Assume by contradiction it is not quasi-supermodular. Then, if $f(I) \geq f\left(\min \left(I, I^{\prime}\right)\right)$, then $f\left(\max \left(I, I^{\prime}\right)\right)<f\left(I^{\prime}\right)$. But that implies that $f\left(\min \left(I, I^{\prime}\right)\right)+$ $f\left(\max \left(I, I^{\prime}\right)\right)<f(I)+f\left(I^{\prime}\right)$, which is a contradiction. Similarly, if $f(I)>f\left(\min \left(I, I^{\prime}\right)\right)$, then $f\left(\max \left(I, I^{\prime}\right)\right) \leq f\left(I^{\prime}\right)$, which again gives us the same contradiction to supermodularity. Therefore, $f$ must be quasi-supermodular. An analogous proof shows that submodularity implies quasi-submodularity. 


\section{A.4 The Simple Plant Location Problem as a Submodular Com- binatorial Discrete Choice Problem}

The Simple Plant Location Problem $(S P L P)$ or Uncapacitated Plant Location Problem $(U P L P)$ refers to a general class of operation research problems concerned with the optimal choice of plants on a topography so as to minimize transportation costs while serving a set of spatially distributed demand points. As the excellent early survey by Krarup and Pruzan (1983) points out, the SPLP is one of the most studied problems in Operations Research, since many other canonical problems can be mapped into it (eg. $p$-Center and $p$-Median

problems), the simple very general structure it provides and lastly the availability of a wide range of effective solution methods. More recent surveys of the literature on the SPLP framework are Cornuéjols et al. (1983), Owen and Daskin (1998) and Verter (2011). While there exists many formulations of the SPLP framework, this paper is going to follow one of the most commonly used ones due to Balinski (1965). In the next section we show how to rewrite the $S P L P$ problem as a combinatorial discrete choice problem with an objective of the form $\Pi: \mathcal{B}^{n} \rightarrow \mathbb{R}$. Next we show that the objective $\Pi$ is submodular which allows the application of $A E R$ to isolate $I^{\star}$. Finally, we show formally how the firm problem in Arkolakis et al. (2013) can be mapped into the SPLP framework.

\section{A.4.1 Writing the Simple Plant Location Problem as a Combinatorial Discrete Choice Problems}

In the baseline version of the $S P L P$, we are given a set $N$ of potential facility locations and a set $D$ demand points. For any location $i \in N$, the fixed cost of opening facility $i$ is $f_{i}$. The cost of serving demand point $j$ from facility $i$ is $c_{i j}$. Note that demand is fixed and there is no intensive margin. The problem is then to find the profit maximizing set of facilities that should be opened. The choice variable is $\lambda_{i j}$ which is 1 if market $j$ is served from location $i$. The $S P L P$ is usually stated as the following minimzation problem, see Balinski (1965):

$$
\begin{array}{r}
\min _{\left\{\lambda_{i j}\right\}_{i \in N, j \in D}} \sum_{i \in N} \sum_{j \in D} c_{i j} \lambda_{i j}+\sum_{i \in N} f_{i} \theta_{i} \\
\text { subject to } \sum_{i \in N} \lambda_{i j}=1 \forall i, j \\
\theta_{i} \geq \lambda_{i j} \forall i, j \\
\lambda_{i j}, \theta_{i} \in\{0,1\} \forall i, j
\end{array}
$$


The constraint on $\lambda_{i j}$ is imposed to ensure no market is served by two facilities. In models with a fixed cost of exporting into a country is automatically satisfied.

This problem can easily be changed into a maximization problem by replacing $c_{i j}$ with $c_{i j}^{\prime}=\max _{i j} c_{i j}-c_{i j}$. Also the choice variable can be expressed as a Boolean vector $I$ of dimension $N$, with the $i$ th entry of 1 corresponding to an open facility in location $i$. For a given plant location choice $I$ the payoff function, $\pi_{k}$, for plant $k$ is defined as follows

$$
\pi_{i}(I)=\sum_{j \in D}\left(\left(\max _{k} c_{k j}^{\prime} I_{k}\right) \mathbb{1}\left(\left(\max _{k} c_{k j}^{\prime} I_{k}\right) \leq c_{i j}^{\prime} I_{i}\right)\right)-f_{i} I_{i}
$$

From this expression, we can get the cumulative profit from all locations:

$$
\Pi(I)=\sum_{i \in N} \pi_{i}(I)=\sum_{i \in N} \sum_{j \in D}\left(\left(\max _{k} c_{k j}^{\prime} I_{k}\right) \mathbb{1}\left(\left(\max _{k} c_{k j}^{\prime} I_{k}\right) \leq c_{i j}^{\prime} I_{i}\right)\right)-f_{i} I_{i}
$$

The decision being made here is to set up a a facility at a specific location. In this case, the decision to set up a plant is synonymous with the decision to pay the fixed cost of a facility, so $I_{k}=\theta_{k}$. The objective is then to choose $I \in\{0,1\}^{n}$ so as to maximize $\Pi(I)$. So the canonical SPLP formulation can be mapped into a combinatorial discrete choice problem with the objective taking the form $\Pi: \mathcal{B}^{n} \rightarrow \mathbb{R}$.

\section{A.4.2 Submodularity of the Overall Profit Function}

The objective function (11) exhibits the submodularity property. Adding an additionl plant will always weakly decrease the number of demand points served by any given previously existing plant making the individual plant profit function (10) decreasing in the number of other plants built. We can then exploit the additive separability property of the overall objective function (11) in combination with lemma 1 to show that the problem is submodular. This allows for the application of the techniques developed in this paper to the solution of problems in the $S P L P$ class.

Lemma 4. The profit function in the simple plant location problem is submodular.

Proof. Let $I$ be such that $I_{i}=1$ and $I_{k}=0 \forall k \neq i$. Consider the profit function of an individual plant in the SPLP problem derived above:

$$
\pi_{i}(I)=\sum_{j \in D}\left(\left(\max _{k} c_{k j}^{\prime} I_{k}\right) \mathbb{1}\left(\left(\max _{k} c_{k j}^{\prime} I_{k}\right) \leq c_{i j}^{\prime} I_{i}\right)\right)-f_{k} I_{k}
$$

Notice that since only plant $i$ is operating, $\max _{k} c_{k j}^{\prime} I_{k}=c_{i j}^{\prime} I_{i}$ trivially since $I_{k}=0 \quad \forall k \neq i$ and $c_{l j}^{\prime} \geq 0 \quad \forall l$ by definition. But then: 


$$
\pi_{i}(I)=\sum_{j \in D} c_{i j}^{\prime} I_{i}-f_{i}
$$

Now consider $I^{\prime}$ so that $I_{i}=I_{s}=1$ and $I_{k}=0 \forall k \neq i, s$. For some $j \in D$ now $\max _{k} c_{k j}^{\prime} I_{k}^{\prime}=$ $c_{s j}^{\prime} I_{s}^{\prime}$. Denote by $D_{i}$ the set of destinations $j$ for which $\max _{k} c_{k j}^{\prime} I_{k}=c_{i j}^{\prime} I_{i}$. Note that $D_{i} \subseteq D$. But then:

$$
\pi_{i}(I)=\sum_{j \in D} c_{i j}^{\prime} I_{i}-f_{i} \geq \sum_{j \in D_{i}} c_{i j}^{\prime} I_{i}-f_{i}=\pi_{i}\left(I^{\prime}\right)
$$

Note that $\pi_{i}\left(I^{i \rightarrow 0}\right)=\pi_{i}\left(I^{\prime, i \rightarrow 0}\right)=0$. The above inequality implies:

$$
\pi_{i}(I)-\pi\left(I^{i \rightarrow 0}\right) \geq \pi_{k}\left(I^{\prime}\right)-\pi\left(I^{\prime, i \rightarrow 0}\right)
$$

Which is the definition of submodularity. Hence $\pi_{k}$ is submodular on the Boolean lattice space. Finally note that $\Pi(I)=\sum_{k} \pi_{k}(I)$. It follows from lemma A.1 that $\Pi(I)$ is submodular on $\mathcal{B}^{n}$.

\section{A.4.3 The Firm Problem in Arkolakis et al. (2013) as a Simple Plant Location Problem}

The firm problem in Arkolakis et al. (2013) can directly be mapped into the formulation in equation (11) using a trick inspired by Hansen and Thisse (1977). We introduce a fictive $N+1$ location in which a plant can be built at zero fixed set up costs, ie. $f_{N+1}=0$. Additionally, goods can be shipped to any demand point $j$ at zero cost so that $c_{N+1} j=0$ for all $j \in D$. Intuitively this is necessary to account for the fact that in Arkolakis et al. (2013) it may be optimal for a firm not to serve a market $j$ from any of its plants if fixed costs are prohibitively high. With this fictive plant in hand we can map the objects from the Arkolakis et al. (2013) problem into objects in the equation (11). In particular $f_{i}$ corresponds to the Arkolakis et al. (2013) fixed cost of setting up a plant in location $i$, while $c_{i j}^{\prime}$ corresponds to the Arkolakis et al. (2013) profit of serving country $j$ from $i$ net of fixed marketing costs. The submodularity of the overall profit function in (11) then implies that the tools of this paper can be employed to identify the profit maximizing strategy vector $I^{\star}$. Deleting its $N+1$ th will then yield the optimal firm strategy in Arkolakis et al. (2013). 